\title{
Blowup of certain analytic solutions of the Hall magnetohydrodynamic equations
}

\author{
Manuel Núñez, ${ }^{1}$ Jorge Álvarez, ${ }^{2}$ and Jesús Rojo ${ }^{2}$ \\ ${ }^{1}$ Departamento de Análisis Matemático, Facultad de Ciencias, Universidad de Valladolid, \\ 47005 Valladolid, Spain \\ ${ }^{2}$ Departamento de Matemática Aplicada, E.T.S. Ingenieros Industriales, Universidad de Valladolid, \\ 47014 Valladolid, Spain
}

(Received 5 March 2008; accepted 25 April 2008; published online 9 June 2008)

\begin{abstract}
A recent analytic solution of the Hall magnetohydrodynamics equations is analyzed. It is shown that its evolution in time depends upon a certain set of inequalities upon the initial values of the velocity and the magnetic field. For most of the cases, both magnitudes will blow up in a finite time. This shows that for keeping the original structure of the solution, energy must be introduced into the system until eventually it cannot hold any longer. (C) 2008 American Institute of Physics.
\end{abstract}

[DOI: 10.1063/1.2930471]

\section{INTRODUCTION}

Hyperbolic topologies for the flow and the magnetic field near a current sheet where magnetic reconnection occurs have a long and distinguished history, starting with the model of Sweet and Parker ${ }^{1-3}$ of two collisional plasma masses. Syrovatskii and co-workers ${ }^{4-6}$ established a solid foundation for the dynamics of this topology, but the problem of how to achieve a fast reconnection rate with it continued to baffle researchers. Petschek's theory of magnetoacoustic shocks ${ }^{7}$ tried to explain how the plasma got away from the sheet fast enough not to throttle the process, and this model was developed by several authors, ${ }^{8-10}$ but the stability of this configuration was questioned by numerical computations. $^{11,12}$ Today it is generally admitted that the single fluid magnetohydrodynamics (MHD) equations fail to describe correctly the process near the current sheets, because of the decoupling of ions and electrons. This has evolved into a well established theory. ${ }^{13-16}$ While it should be necessary to study the two-fluid MHD equations to mathematically analyze this problem, an intermediate step is to merely add the Hall term to the induction equation, which describes the essential mechanisms in a rather satisfactory manner. The striking part is that there exists a spatial geometry, where the coefficients depend only on time, that provides exact solutions of this Hall MHD system. This should be credited to Shivamoggi. ${ }^{17}$ Specific analytic solutions have always played a prominent role in the study of magnetic reconnection; they provide insights on the detailed behavior of the magnetic field out of reach of any phenomenological argument such as those of Sweet and Parker. Thus, a class of analytic solutions containing those of Petschek, ${ }^{7}$ Sonnerup, ${ }^{9}$ and Yeh and Axford ${ }^{10}$ may be found in Ref. 18; the models of Syrovatskii et $a l^{5,6}$ are also represented by analytic functions. Refined analytic solutions, and the phenomena they describe, are analyzed in Refs. 19 and 20. Hall magnetohydrodynamics is not so rich in analytic solutions. Craig and Watson $^{21}$ provide one, but the analysis of the connection between different subdomains is asymptotic and relies on properties of special functions. This is one of the reasons why the simple solution of Shivamoggi is worth consideration. We will find that there exists a range of possibilities depending upon the initial conditions of the magnetic field and the velocity, but with the exception of two cases, both magnitudes will blow up in a finite time. To prove that solutions exist for only a finite time would be a major result, e.g., for the Navier-Stokes or the MHD and Hall MHD equations, but our result unfortunately does not throw any light on these problems. Everything depends on the boundary conditions; these are crucial in the study of magnetic reconnection ${ }^{22-25}$ and in fact in every evolutionary system. When these conditions preclude an input of (kinetic or magnetic) energy from the outside of the domain, the total energy is conserved or dissipated by viscous losses and Ohmic heating; what is not known yet in the classic problems of fluid mechanics is if the solution loses regularity at a finite time, whereas what we find here is a genuine explosion; magnetic field and velocity go to infinity. This proves that to keep the original analytic form of the solution, energy must be fed into the system until eventually it cannot be absorbed. This statement must be clarified. Although the solutions within this paper may be defined in the whole space, they would have infinite energy there. Instead we will assume that both are defined only in a fixed bounded domain $\Omega$, so that both kinetic and magnetic energies are finite at $t=0$. The form of the solution obviously determines how the variables behave at the boundary of $\Omega$, which means that the boundary conditions should be specified so that the solution satisfies them, and they must change in time following the evolution of the solution. The fact that the energy blows up in a finite time means that the evolving boundary conditions correspond to an input of energy within $\Omega$. The point is that to make boundary values slave to a particular solution, and not vice versa, may mean the introduction of energy within the domain until it eventually cannot hold it. To a certain extent this occurs for many analytic solutions of MHD problems, but in this case the consequences are catastrophic. Thus, while the interest of this configuration is limited from the point of view of physically 
realistic magnetic reconnection, it is conceptually interesting both in its structure and in its consequences.

\section{GOVERNING EQUATIONS AND STRUCTURE OF THE SOLUTIONS}

While the Hall MHD equations are inherently threedimensional, solutions may be found depending only on two spatial variables. ${ }^{21}$ The whole system, properly normalized for an incompressible flow, is

$$
\begin{aligned}
& \frac{\partial \mathbf{u}}{\partial t}+\mathbf{u} \cdot \nabla \mathbf{u}=\nu \Delta \mathbf{u}+\mathbf{J} \times \mathbf{B}-\nabla P, \\
& \frac{\partial \mathbf{B}}{\partial t}=\nabla \times(\mathbf{u} \times \mathbf{B})+\eta \Delta \mathbf{B}-h \boldsymbol{\nabla} \times(\mathbf{J} \times \mathbf{B}), \\
& \boldsymbol{\nabla} \cdot \mathbf{u}=\boldsymbol{\nabla} \cdot \mathbf{B}=0 .
\end{aligned}
$$

$\mathbf{u}$ represents the velocity, $\mathbf{B}$ the magnetic field, $\mathbf{J}=\boldsymbol{\nabla} \times \mathbf{B}$ the current density, $\nu$ the viscosity, $\eta$ the resistivity, and $h$ the Hall coefficient. If we assume that all the magnitudes depend only on the coordinates $(x, y)$, we may write the magnetic field in terms of a flux function $\psi$ as

$$
\mathbf{B}=\left(\boldsymbol{\nabla} \psi \times \mathbf{e}_{3}\right)+b \mathbf{e}_{3}=\left(\frac{\partial \psi}{\partial y},-\frac{\partial \psi}{\partial x}, b\right) .
$$

The same is true for the velocity, but since we will impose a fixed stream function later, let us simply write

$$
\mathbf{u}=\left(v_{1}, v_{2}, w\right)=(\mathbf{v}, w) .
$$

Then

$$
\begin{aligned}
& \mathbf{J}=\left(\frac{\partial b}{\partial y},-\frac{\partial b}{\partial x},-\Delta \psi\right), \\
& \mathbf{J} \times \mathbf{B}=-\frac{1}{2} \boldsymbol{\nabla} b^{2}-\Delta \psi \nabla \psi+(0,0,[b, \psi]),
\end{aligned}
$$

where

$$
\begin{aligned}
& {[f, g]=\frac{\partial f}{\partial x} \frac{\partial g}{\partial y}-\frac{\partial f}{\partial y} \frac{\partial g}{\partial x},} \\
& \boldsymbol{\nabla} \times(\mathbf{J} \times \mathbf{B})=-\boldsymbol{\nabla}(\Delta \psi) \times \boldsymbol{\nabla} \psi+(\boldsymbol{\nabla}[b, \psi]) \times \mathbf{e}_{3} .
\end{aligned}
$$

The first term in Eq. (8) lies along the z-axis and the second one in the $x, y$-plane. Finally,

$$
\mathbf{u} \times \mathbf{B}=w \boldsymbol{\nabla} \psi+b\left(v_{2},-v_{1}, 0\right)-(\mathbf{v} \cdot \boldsymbol{\nabla} \psi) \mathbf{e}_{3} .
$$

Therefore,

$$
\boldsymbol{\nabla} \times(\mathbf{u} \times \mathbf{B})=([w, \psi]-\mathbf{v} \cdot \boldsymbol{\nabla} b) \mathbf{e}_{3}-\boldsymbol{\nabla} \times\left((\mathbf{v} \cdot \boldsymbol{\nabla} \psi) \mathbf{e}_{3}\right) .
$$

With this we may decompose the momentum equation [Eq. (1)] into

$$
\frac{\partial \mathbf{v}}{\partial t}+\mathbf{v} \cdot \nabla \mathbf{v}=\nu \Delta \mathbf{v}-\nabla\left(P+(1 / 2) b^{2}\right)-\Delta \psi \nabla \psi,
$$

$$
\frac{\partial w}{\partial t}+\mathbf{v} \cdot \nabla w=\nu \Delta w+[b, \psi]
$$

and the induction equation [Eq. (2)] into the vertical component

$$
\frac{\partial b}{\partial t}+\mathbf{v} \cdot \nabla b=\eta \Delta b+[w, \psi]+h[\Delta \psi, \psi]
$$

and the horizontal component

$$
\boldsymbol{\nabla} \times\left[\left(\frac{\partial \psi}{\partial t}+\mathbf{v} \cdot \boldsymbol{\nabla} \psi-\eta \Delta \psi+h[b, \psi]\right) \mathbf{e}_{3}\right]=\mathbf{0} .
$$

Uncurling this last equation,

$$
\frac{\partial \psi}{\partial t}+\mathbf{v} \cdot \nabla \psi-\eta \Delta \psi+h[b, \psi]=g,
$$

where $g$ is a function of time (assuming the plane domain is connected). $g$ could be determined by additional constraints, but we will leave it as general as possible.

Equations (11)-(14) form the basic system. Now we define the ansatz for the functions $\mathbf{v}, \psi, w, b$, and $P$,

$$
\mathbf{v}=(-\dot{F}(t) x, \dot{F}(t) y),
$$

where $F$ is a function of time. The reason why we define $\mathbf{v}$ in terms of $\dot{F}$ is to simplify later calculations: We will take (as we obviously can) $F(0)=0$. Notice that the projections in the plane of the streamlines are always hyperbolas $x y=$ const. We set

$$
\psi=G(t) x^{2}-H(t) y^{2}+\psi_{0}(t)
$$

$G$ and $H$ are always positive. $\psi_{0}$ has no influence upon the magnetic field, but it will be useful to close the equations,

$$
w=-\frac{1}{h} \psi+w_{0}(t)
$$

This is the only point where the existence of a Hall coefficient $h \neq 0$ is crucial. Finally

$$
b=k x y,
$$

where $k$ is a constant independent of time, and

$$
P=L(t)\left(x^{2}+y^{2}\right)-\frac{1}{2} b^{2} .
$$

From this ansatz we easily deduce the following expressions:

$$
\frac{\partial \mathbf{v}}{\partial t}=(-\ddot{F} x, \ddot{F} y)
$$

$$
\mathbf{v} \cdot \nabla \mathbf{v}=\left(\dot{F}^{2} x, \dot{F}^{2} y\right)
$$

$\Delta \mathbf{v}=\mathbf{0}$,

$$
\nabla \psi=2(G x,-H y),
$$

$$
\frac{\partial \psi}{\partial t}=\dot{G} x^{2}-\dot{H} y^{2}+\dot{\psi}_{0},
$$

$\mathbf{v} \cdot \nabla \psi=-2 \dot{F}\left(G x^{2}+H y^{2}\right)$, 


$$
\begin{aligned}
& \Delta \psi=2(G-H), \\
& \nabla\left(P+(1 / 2) b^{2}\right)=2 L(x, y), \\
& {[b, \psi]=-2 k G x^{2}-2 k H y^{2},} \\
& {[\Delta \psi, \psi]=0 .}
\end{aligned}
$$

Therefore Eq. (11) becomes

$$
\ddot{F}(-x, y)+\dot{F}^{2}(x, y)=-2 L(x, y)-4(G-H)(G x,-H y) .
$$

That is,

$$
\begin{gathered}
-\ddot{F}+\dot{F}^{2}=-2 L-4(G-H) G, \\
\ddot{F}+\dot{F}^{2}=-2 L+4(G-H) H .
\end{gathered}
$$

By adding and subtracting,

$$
\begin{aligned}
& \ddot{F}=2\left(G^{2}-H^{2}\right), \\
& \dot{F}^{2}=-2 L-2(G-H)^{2} .
\end{aligned}
$$

If we are able to write $G$ and $H$ as functions of $F$ and solve Eq. (23), we may use afterwards Eq. (24) to define $L$. Hence Eq. (11) may be reduced to Eq. (23).

It is convenient now to turn to Eq. (14). It becomes

$$
\begin{aligned}
\dot{G} x^{2}-\dot{H} y^{2}+\dot{\psi}_{0}-2 \dot{F} G x^{2}-2 \dot{F} H y^{2}-2 h k G x^{2}-2 h k H y^{2} \\
\quad-2 \eta(G-H)=g .
\end{aligned}
$$

That is,

$$
\begin{aligned}
& \dot{G}-2 \dot{F} G-2 h k G=0, \\
& -\dot{H}-2 \dot{F} H-2 h k H=0, \\
& \dot{\psi}_{0}-2 \eta(G-H)=g .
\end{aligned}
$$

Equation (26) has the solution

$$
G(t)=G(0) \exp (2 F(t)+2 h k t),
$$

whereas Eq. (27) implies

$$
H(t)=H(0) \exp (-2 F(t)-2 h k t) .
$$

Once $G$ and $H$ are known, Eq. (28) simply becomes the definition of $\psi_{0}$.

Taking Eqs. (29) and (30) to Eq. (23), we find the main equation

$$
\begin{aligned}
\ddot{F}= & 2 G(0)^{2} \exp (4 F(t)+4 h k t)-2 H(0)^{2} \\
& \times \exp (-4 F(t)-4 h k t) .
\end{aligned}
$$

Once Eq. (31) is solved for $F(0)=0, \dot{F}(0)$ arbitrary, we may use Eqs. (23), (29), and (30) to obtain a solution for Eqs. (11) and (14). It remains to be seen that Eqs. (12) and (13) are satisfied as well. Given the expression (17) of $w$,

$$
\frac{\partial w}{\partial t}+\mathbf{v} \cdot \boldsymbol{\nabla} w-[b, \psi]=-\frac{1}{h}\left(\frac{\partial \psi}{\partial t}+\mathbf{v} \cdot \nabla \psi\right)-[b, \psi]+\dot{w}_{0},
$$

which, since $\psi$ satisfies Eq. (14), yields

$$
\frac{\partial w}{\partial t}+\mathbf{v} \cdot \nabla w-[b, \psi]=\dot{w}_{0}-\frac{1}{h} g-\frac{2 \eta}{h}(G-H) .
$$

This needs to be equal to the remaining term in Eq. (12), $\nu \Delta w=-(\nu / h) \Delta \psi=-(2 \nu / h)(G-H)$. Hence

$$
\dot{w}_{0}=\frac{1}{h} g+\frac{2 \eta}{h}(G-H)-\frac{2 \nu}{h}(G-H) .
$$

Defining $w_{0}$ in this way, Eq. (12) is automatically satisfied.

Let us see that Eq. (13) holds trivially. We have $[\Delta \psi, \psi]=[w, \psi]=\Delta b=\dot{b}=0$. Finally,

$$
\mathbf{v} \cdot \nabla b=-k \dot{F} x y+k \dot{F} x y=0 .
$$

Therefore the whole system reduces to Eq. (31), an ordinary differential equation on the flow function $F$.

\section{EVOLUTION OF THE FLOW FUNCTION}

Define

$$
S(t)=4 F(t)+4 h k t .
$$

Then $S$ satisfies

$$
\begin{aligned}
& \ddot{S}(t)=8 G(0)^{2} e^{S(t)}-8 H(0)^{2} e^{-S(t)}, \\
& S(0)=0 .
\end{aligned}
$$

Equation (36) admits a first integral. Multiplying both sides by $\dot{S}(t)$,

$$
\frac{d}{d t}\left(\frac{1}{2} \dot{S}(t)^{2}\right)=\frac{d}{d t}\left(8 G(0)^{2} e^{S(t)}+8 H(0)^{2} e^{-S(t)}\right) .
$$

Hence the difference between the functions on the left- and the right-hand side is a constant, which may be found by considering the value $\dot{S}(0)$. If we denote

$$
16 G(0)^{2}=\alpha, \quad 16 H(0)^{2}=\beta, \quad \dot{S}(0)=\gamma,
$$

we obtain

$$
\dot{S}(t)^{2}=\alpha e^{S(t)}+\beta e^{-S(t)}-\alpha-\beta+\gamma^{2} .
$$

With this notation, Eqs. (29) and (30) may be written as

$$
\begin{aligned}
& G(t)=G(0) \exp \left(\frac{1}{2} S(t)\right), \\
& H(t)=H(0) \exp \left(-\frac{1}{2} S(t)\right) .
\end{aligned}
$$

To analyze the behavior of the solution to Eq. (38), let us consider the function 


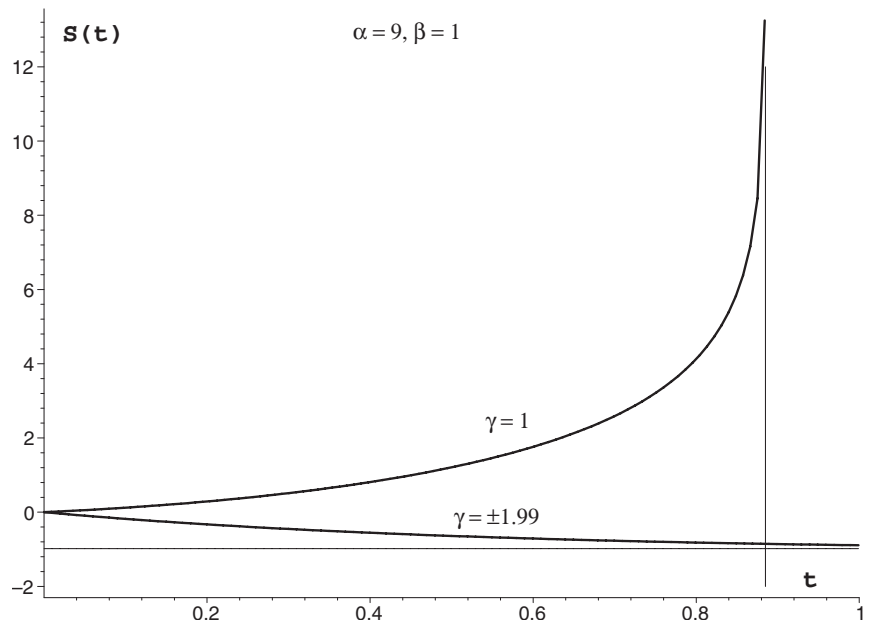

FIG. 1. Evolution of the flow function in case 1.

$$
f(x)=\alpha x+\frac{\beta}{x}+\gamma^{2}-\alpha-\beta,
$$

defined for $x>0$. It tends to infinity both when $x \rightarrow 0$ and $x \rightarrow \infty$. Its minimum lies at $x_{0}=\sqrt{\beta / \alpha}$, and the value of $f$ at this point is

$$
f\left(x_{0}\right)=\gamma^{2}-(\sqrt{\alpha}-\sqrt{\beta})^{2} .
$$

Therefore, for $|\gamma|>|\sqrt{\alpha}-\sqrt{\beta}|, f$ has no zeroes and is bounded below by $f\left(x_{0}\right)>0$. For $|\gamma|=|\sqrt{\alpha}-\sqrt{\beta}|, f$ has precisely one zero at $x_{0}$, whereas for $|\gamma|<|\sqrt{\alpha}-\sqrt{\beta}|$, it has two. In this case these zeroes are

$$
z_{ \pm}=\frac{1}{2 \alpha}\left[\alpha+\beta-\gamma^{2} \pm \sqrt{\left(\alpha+\beta-\gamma^{2}\right)^{2}-4 \alpha \beta}\right]
$$

Let us consider the case where both $z_{-}$and $z_{+}$are real and different. Since the right-hand side of Eq. (38) must be positive for any solution, $\exp [S(t)]$ cannot lie in the interval $\left(z_{-}, z_{+}\right)$. The points $z_{ \pm}$correspond to stationary solutions of Eq. (38); these satisfy $S(0)=\log \left(z_{ \pm}\right)$and obviously $\dot{S}(0)=0$. Since in our case $\dot{S}(0)=\gamma$, unless $\gamma=0$ the solution cannot be constant. If $\gamma=0, z_{ \pm}=(1, \beta / \alpha)$; thus $\gamma=0$ corresponds to the stationary solution $S(t)=0$. [Recall that from Eq. (35), $S(0)=0$, so that $S(t)=\log (\beta / \alpha)$ is not admissible unless $\alpha=\beta$.] For the nontrivial case $\gamma \neq 0$, the right-hand side of Eq. (38) equals $\gamma^{2}>0$, so that it does not lie within $\left(z_{-}, z_{+}\right)$ (which, we recall, may be empty). Taking the square root of Eq. (38),

$$
\dot{S}(t)=\left[\alpha e^{S(t)}+\beta e^{-S(t)}+\gamma^{2}-\alpha-\beta\right]^{1 / 2},
$$

we obtain the final differential equation, but we need to know the appropriate sign of the root. Since $\dot{S}(0)=\gamma$, this sign must be positive if $\gamma>0$, negative if $\gamma<0$. For $|\gamma|>|\sqrt{\alpha}-\sqrt{\beta}|$, this is the only datum we need to predict the evolution of the solution. If, however, $\left(z_{-}, z_{+}\right)$is a real interval, there are four possible alternatives: $\gamma>0,1>z_{+}$, $\gamma<0,1>z_{+}, \quad \gamma>0,1<z_{-}$, and $\gamma<0,1<z_{-}$. Let us study each possibility in turn.

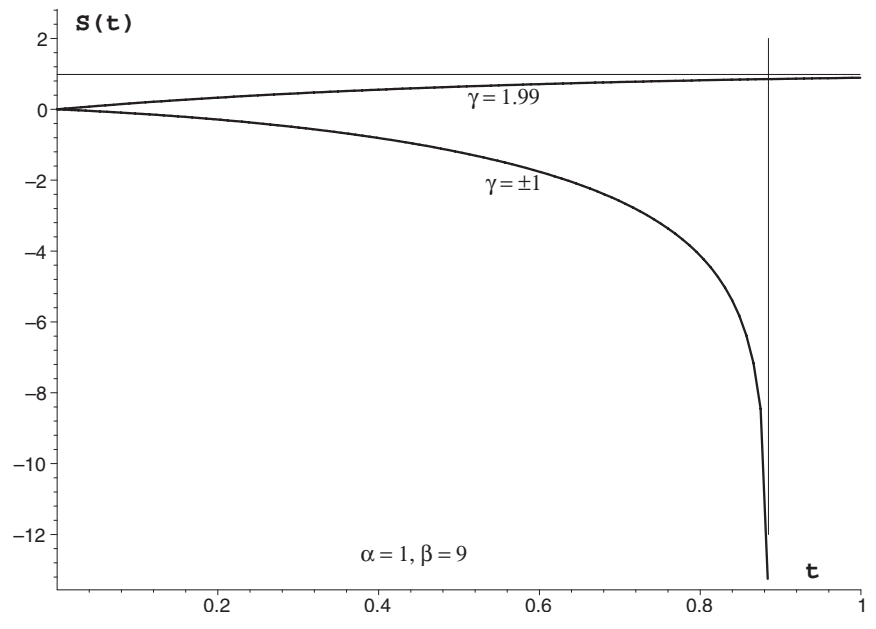

FIG. 2. Evolution of the flow function in case 2 .

\section{A. Case 1: $z_{+}<1$}

Consider first the case $\gamma>0$. Then $\dot{S}(t)>0$ and $S(t)$ grows; consequently so does $\exp [S(t)]$ and $f[\exp (S(t)]$, since $f$ [given by Eq. (41)] increases from $z_{+}$to $\infty$. Thus $S(t)$ always increases; let us see that in fact it blows up in a finite time. Since $t \rightarrow S(t)$ is an admissible change of variable from $t=0$ onwards, writing Eq. (44) as

$$
\frac{\dot{S}(t)}{\left[\alpha e^{S(t)}+\beta e^{S(t)}+\gamma^{2}-\alpha-\beta\right]^{1 / 2}}=1,
$$

and integrating in time,

$$
\int_{0}^{t_{0}} \frac{\dot{S}(t) d t}{\left[\alpha e^{S(t)}+\beta e^{S(t)}+\gamma^{2}-\alpha-\beta\right]^{1 / 2}}=t_{0},
$$

i.e.,

$$
\int_{0}^{S\left(t_{0}\right)} \frac{d r}{\left[\alpha e^{r}+\beta e^{-r}+\gamma^{2}-\alpha-\beta\right]^{1 / 2}}=t_{0} .
$$

Since we know a priori that the denominator is always larger than $\gamma>0$, and the integrand behaves at $r=\infty$ like $\exp (-r / 2)$, which has a finite integral upon $[0, \infty)$,

$$
\int_{0}^{\infty} \frac{d r}{\left[\alpha e^{r}+\beta e^{-r}+\gamma^{2}-\alpha-\beta\right]^{1 / 2}}=t_{\infty}<\infty .
$$

Since general theorems prove that the solution of an ordinary differential equation may be continued for as long as it remains bounded, we conclude that the interval of existence of $S(t)$ is precisely $\left[0, t_{\infty}\right)$, and $\lim _{t \rightarrow t_{\infty}} S(t)=\infty$.

Assume now that $z_{+}<1, \gamma<0$. Then we must take the minus sign in the square root at Eq. (44), and therefore $\dot{S}(t)<0$ and $S$ decreases for as long as $f(\exp (S(t))>0$. This means that $\lim _{t \rightarrow \infty} \exp (S(t))=z_{+}, \lim _{t \rightarrow \infty} S(t)=\log z_{+}$. In this case the solution remains bounded and therefore exists for all time; the asymptotic limit $\log z_{+}$is never reached in a finite time. These two alternatives are studied in Fig. 1 for specific values of $\alpha, \beta$, and $\gamma$. Equation (44) has been integrated numerically using a Burlirsch-Stoer rational extrapolation method. The value of $t_{\infty}$ is tabulated independently by evalu- 


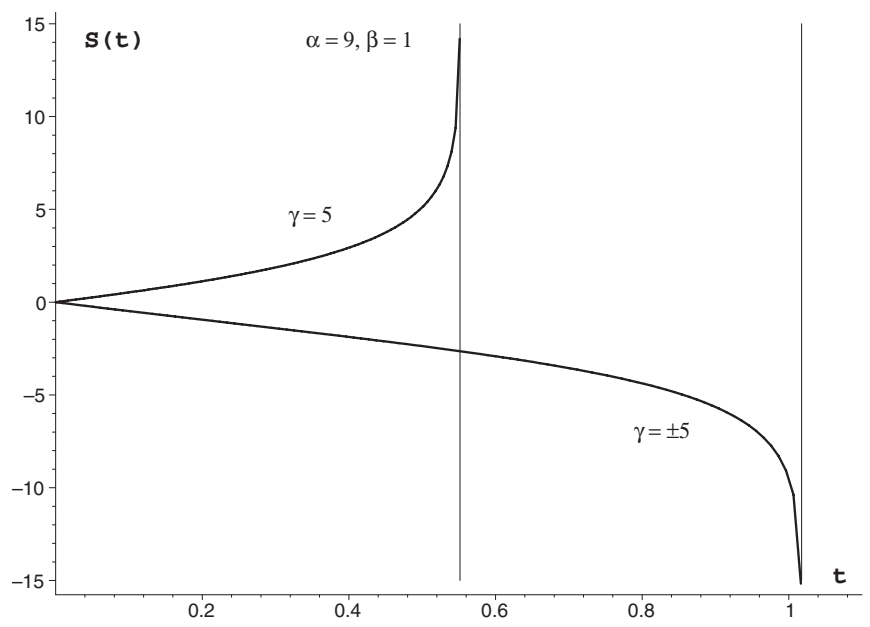

FIG. 3. Evolution of the flow function in case 3 .

ating numerically the integral Eq. (48); its location corresponds to the vertical line. We see that it coincides precisely with the point where the solution blows up.

\section{B. Case 2: $z_{-}>1$}

The situation is symmetric when $1<z_{-}$. Now $\gamma>0$ means that $S(t)$ increases for as long as $f(\exp (S(t))>0$, i.e., $\lim _{t \rightarrow \infty} S(t)=\log z_{-}$. On the other hand, $\gamma<0$ means that $S(t)$ and $\exp (S(t))$ decreases in time, and $f(\exp (S(t)))$ grows when $\exp (S(t))$ decreases to zero. Thus the rate of decreasing grows in time, and in analogy to the first case, in the time

$$
\int_{-\infty}^{0} \frac{d r}{\left|\left[\alpha e^{r}+\beta e^{-r}+\gamma^{2}-\alpha-\beta\right]^{1 / 2}\right|}=t_{-\infty}<\infty,
$$

the solution must blow up; only this time the limit of $S(t)$ is $-\infty$. We have written the absolute value to emphasize that the integrand is always positive. Both alternatives are represented in Fig. 2 for certain values of the parameters.

\section{Case 3: $z_{-}, z_{+}$are not real numbers}

This case is the simplest of all: When $|\gamma|>|\sqrt{\alpha}-\sqrt{\beta}|$ and the interval $\left[z_{-}, z_{+}\right]$vanishes, only the blowup possibility exists. For $\gamma>0$,

$$
\lim _{t \rightarrow t_{\infty}} S(t)=\infty,
$$

whereas for $\gamma<0$,

$$
\lim _{t \rightarrow t_{-\infty}} S(t)=-\infty \text {. }
$$

For $\gamma>0$ the solution increases and eventually tends to $\infty$ in a finite time given by Eq. (50); while for $\gamma<0$ it tends to $-\infty$ at the time given by Eq. (51). There is no problem with the chance that the denominator of both integrals may tend to zero, since it is bounded below by a positive constant. This is illustrated in Fig. 3.

Since the limits of $G(t)$ and $H(t)$ are given by Eqs. (39) and (40), we see that certain inequalities between the original values of the velocity and the magnetic field determine the evolution of the system. With the exception of two possible cases, one of the plane components of the magnetic field blows up in a finite time. Since in the case of blowup $S(t)$ either increases or decreases monotonically, $\dot{S}(t)$ blows up in the same time interval. $S$ is defined by Eq. (35), so that $\dot{F}$ blows up at the same time. When $S$ has a finite limit, $\dot{S}(t)$ tends to zero when $t \rightarrow \infty$ and therefore $\dot{F}(t)$ tends to $-h k$. Therefore the velocity blows up when the magnetic field does, and if there is no blowup and the field tends to a finite limit, the plane velocity tends to a constant depending on the Hall coefficient.

\section{CONCLUSIONS}

A set of analytic expressions proposed by Shivamoggi has been shown to provide a solution of the Hall MHD system. These expressions are polynomials in the space variables, while the coefficients depend on time to conform with the evolution determined by the original set of equations. It is shown here that all these equations may be reduced to a single ordinary differential one for a single scalar function. The form of this equation depends upon the initial values of velocity and magnetic field, and its analysis shows that in all but two possible sets of inequalities for these initial values, it tends to infinity in a finite time. In the remaining two cases the magnetic field and the velocity have asymptotic limits. When blowup exists, the velocity and the magnetic field tend to infinity. Since the Hall MHD system conserves the energy (except by dissipative losses) for boundary conditions precluding an input of energy from the outside, we conclude that to keep the structure of the solutions, energy must have been introduced into the system until it blows up. Hence, while this solution is not realistic from a strictly physical viewpoint, both its form and its behavior are extremely illuminating as they show how artificial boundary conditions that are arbitrarily set to keep a certain kinetic and magnetic geometry may be a source of major unphysical consequences.

${ }^{1}$ P. A. Sweet, Nuovo Cimento 8, 188 (1958).

${ }^{2}$ E. N. Parker, J. Geophys. Res. 62, 509, DOI: 10.1029/JZ062i004p00509 (1957).

${ }^{3}$ E. N. Parker, Astrophys. J., Suppl. 8, 177 (1963).

${ }^{4}$ V. S. Imshennik and S. I. Syrovatskii, Sov. Phys. JETP 25, 656 (1967).

${ }^{5}$ S. I. Syrovatskii, Sov. Phys. JETP 33, 933 (1971).

${ }^{6}$ S. I. Syrovatskii and S. V. Bulanov, Annu. Rev. Astron. Astrophys. 19, 163 (1981).

${ }^{7}$ H. E. Petschek, "Magnetic field annihilation," in Physics of Solar Flares, edited by W. H. Ness (NASA SP-50, Washington, D.C., 1964), pp. 425-439.

${ }^{8}$ W. I. Axford, Space Sci. Rev. 7, 149 (1967).

${ }^{9}$ B. U. O. Sonnerup, J. Plasma Phys. 4, 161 (1970).

${ }^{10}$ T. Yeh and W. I. Axford, J. Plasma Phys. 4, 207 (1970).

${ }^{11}$ D. Biskamp, Phys. Fluids 29, 1520 (1986).

${ }^{12}$ D. Biskamp, Nonlinear Magnetohydrodynamics (Cambridge University Press, Cambridge, 1993).

${ }^{13}$ J. F. Drake, D. Biskamp, and A. Zeiler, Geophys. Res. Lett. 24, 2921, DOI: 10.1029/97GL52961 (1997).

${ }^{14}$ M. A. Shay, J. F. Drake, B. N. Rogers, and R. E. Denton, J. Geophys. Res. 106, 3759, DOI: 10.1029/1999JA001007 (2001).

${ }^{15}$ B. N. Rogers, R. E. Denton, J. F. Drake, and M. A. Shay, Phys. Rev. Lett. 87, 195004 (2001).

${ }^{16}$ D. Biskamp, Magnetic Reconnection in Plasmas (Cambridge University Press, Cambridge, 2000). 
${ }^{17}$ B. K. Shivamoggi, Phys. Lett. A 258, 131 (1999).

${ }^{18}$ E. R. Priest and T. G. Forbes, J. Geophys. Res. 91, 5579, DOI: 10.1029/ JA091iA05p05579 (1986).

${ }^{19}$ V. S. Titov and E. R. Priest, J. Fluid Mech. 348, 327 (1997).

${ }^{20}$ E. R. Priest and V. S. Titov, Philos. Trans. R. Soc. London, Ser. A 354, 2951 (1996).

${ }^{21}$ I. J. D. Craig and P. G. Watson, Sol. Phys. 214, 131 (2003).
${ }^{22}$ M. Yan, L. C. Lee, and E. R. Priest, J. Geophys. Res. 97, 8277, DOI: 10.1029/92JA00170 (1992).

${ }^{23}$ E. R. Priest and T. G. Forbes, J. Geophys. Res. 97, 16757, DOI: 10.1029/ 92JA01146 (1992).

${ }^{24}$ E. R. Priest and T. G. Forbes, Magnetic Reconnection: MHD Theory and Applications (Cambridge University Press, Cambridge, 2000).

${ }^{25}$ M. Núñez, Fluid Dyn. Res. 39, 447 (2007). 